\title{
Strong Cluster Properties for Classical Systems with Finite Range Interaction
}

\author{
M. Duneau \\ Centre de Physique Théorique de l'Ecole Polytechnique, Paris, France \\ «Equipe de Recherche Associée au C.N.R.S.» \\ D. Iagolnitzer \\ Service de Physique Théorique, Centre d'Etudes Nucléaires de Saclay, \\ Gif-sur-Yvette, France \\ B. Souillard \\ Centre de Physique Théorique de l'Ecole Polytechnique, Paris, France \\ «Equipe de Recherche Associée au C.N.R.S.»
}

Received October 25, 1973

\begin{abstract}
In a previous paper, "strong" decrease properties of the truncated correlation functions, taking into account the separation of all particles with respect to each other, have been presented and discussed.

In this paper, we prove these properties for finite range interactions in various situations, in particular

i) at low activity for lattice and continuous systems,

ii) at arbitrary activity and high temperature for lattice systems,

iii) at $\operatorname{Re} H \neq 0, \beta$ arbitrary and at $H=0$ for appropriate temperatures in the case of ferromagnets.

We also give some general results, in particular an equivalence, on the links between analyticity and strong cluster properties of the truncated correlation functions.
\end{abstract}

\section{Introduction}

\section{A. Strong Cluster Properties of the Truncated Correlations for Finite Range Interactions}

In [1], strong cluster properties of the truncated ${ }^{1}$ correlation functions, which "take into account the separation of all particles with respect to each other", have been presented and discussed. For simplicity we first consider lattice systems, with two-body potentials, the case of classical continuous systems being treated in Section VI.

The strong cluster properties have then been stated in the form:

$$
\left|\varrho_{\Lambda}^{T}\left(x_{1}, \ldots, x_{N}\right)\right| \leqq \sum_{\mathscr{T}} \prod_{l \in \mathscr{T}} u\left(d_{l}\right)
$$

\footnotetext{
1 Also called connected parts or cluster functions of the correlations.
} 
where the sum $\Sigma$ runs over all connected trees $\mathscr{T}$ (i.e. graphs without closed loops) of $(N-1)$ lines joining the points $x_{1}, \ldots, x_{N}, d_{l}$ is the length of line $l$ and $u$ is a positive function, decreasing at infinity, which depends on the interaction $\phi$, the reciprocal temperature $\beta$ and the activity $z$.

It was proved that, when $u$ is integrable, these bounds yield the existence and analyticity of all (infinite volume) correlation functions with respect to $z$ at all points $\left(\beta_{0}, z_{0}\right)$ where they hold.

If the function $u$ is of the form $\hat{C} \cdot e^{-\hat{\chi} \cdot d}$, (where $\hat{C}$ and $\hat{\chi}$ depend on $\phi$, $\beta$ and $z$ ), (1) takes the form:

$$
\left|\varrho_{\Lambda}^{T}\left(x_{1}, \ldots, x_{N}\right)\right| \leqq \hat{C}^{N} \sum_{\mathscr{T}} e^{-\hat{\chi} \cdot \hat{L}_{\mathscr{T}}(X)}
$$

where $\hat{L}_{\mathscr{T}}(X)$ is the length of tree $\mathscr{T}$, i.e. the sum of the lengths of its lines.

In the present paper, we are going to prove, in the case of finite range interactions and under appropriate conditions, bounds which are slightly better: the term $\sum_{\mathscr{T}} e^{-\hat{x} \cdot \hat{L}_{\mathscr{T}}(X)}$ will be replaced by the unique term $e^{-\hat{\chi} \cdot \hat{L}(X)}$, with $\hat{L}(X)=\operatorname{Inf}_{\mathscr{T}} \hat{L}_{\mathscr{T}}(X)$ when the points $x_{1}, \ldots, x_{N}$ are all different from each other; when $x_{1}, \ldots, x_{N}$ occupy only $p$ different positions occuring respectively $N_{1}, \ldots, N_{p}$ times, the term obtained is $N_{1} ! \ldots N_{p} ! e^{-\hat{\chi} \cdot \hat{L}(X)}$. The corresponding bounds are:

$$
\left|\varrho_{\Lambda}^{T}\left(x_{1}, \ldots, x_{N}\right)\right| \leqq \hat{C}^{N} N_{1} ! \ldots N_{p} ! e^{-\hat{\chi} \cdot \hat{L}(X)} .
$$

[The right-hand side of (3) is indeed always smaller than the right hand side of (2) since the number of trees joining $N_{i}$ points is larger than $N_{i}$ !.]

Finally it is also useful, as in [1], to introduce the minimal length $L(X)$ of all connected trees joining $x_{1}, \ldots, x_{N}$ and possibly arbitrary vertices $y_{1}, \ldots, y_{k}$. An example of the lengths $\hat{L}(X)$ and $L(X)$ is shown in Fig. 1 for $N=3$ :

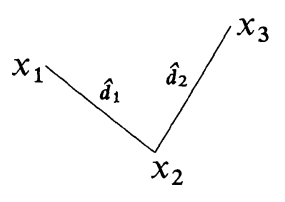

$$
\hat{L}(X)=\hat{d}_{1}+\hat{d}_{2}
$$

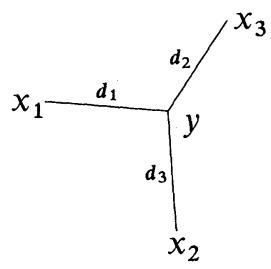

$L(X)=d_{1}+d_{2}+d_{3}$

Fig. 1

In view of the inequality $\frac{1}{2} \hat{L}(X) \leqq L(X) \leqq \hat{L}(X)$ which holds for all $X$ and $N$ (see [1]), it is equivalent to prove (3) or:

$$
\left|\varrho_{\Lambda}^{T}\left(x_{1}, \ldots, x_{N}\right)\right| \leqq C^{N} N_{1} ! \ldots N_{p} ! e^{-\chi \cdot L(X)}
$$

where $C$ and $\chi$ are independent of $\Lambda, X$ and $N$. 
This is the form in which we are going to derive the bounds for lattice gases, analogous bounds being derived in Section VI for continuous systems.

Remark. Since the bounds (3), (4) are slightly better than (1), (2), they also provide the analyticity of all correlation functions at all points $\left(\beta_{0}, z_{0}\right)$ where they hold. We recall that decrease factors which would not take into account the separation of all particles with respect to each other, such as those described in Subsection B (or constants growing "faster" than $C^{N} N_{1}$ ! ... $N_{p}$ ! with $N$ ) would prevent one from deriving analyticity.

However, in the various cases where the bounds are derived in the present paper, the analyticity with respect to $z$ is already known or assumed (see Subsection C) and therefore no new information about analyticity is obtained. But it seems to us that the strong cluster properties have their own physical interest. Besides, the combined results of [1] and of the present paper allow (in a sense made precise later) to obtain an equivalence between analyticity and strong cluster properties which also seems to be an interesting result. Finally, the fact that the strong cluster properties have been proved in certain domains reinforces the underlying physical ideas and work is in progress to prove these properties in more general cases.

\section{B. Previous Results about Cluster Properties}

The results previously proved about the decrease properties of the truncated correlations are, as far as we know, weaker than (1) to (4); in the case of finite range interactions, let us mention:

i) Those derived from factorization properties of the (non-truncated) correlation functions when two (or more) clusters of points are separated from each other, give at best:

$$
\left|\varrho_{\Lambda}^{T}(X)\right|<D_{N} e^{-\chi \cdot \operatorname{Max} d\left(X_{1}, X_{2}\right)}
$$

where $X_{1}, X_{2}$ is any partition of $X$ in two clusters, $d\left(X_{1}, X_{2}\right)$ is the distance between $X_{1}$ and $X_{2}$, and where $D_{N} \geqq N ! C^{N}$.

Property (5) has been derived in [2] from the decrease of the twopoint function when there is a gap in the spectrum of the transfer matrix and when FKG inequalities are valid.

We note that besides the fact that $D_{N}$ is worse than $C^{N} N_{1} ! \ldots N_{p}$ ! the decrease $e^{-\chi x_{1}, x_{2}}$ is also much weaker than $e^{-\chi L(X)}:$ for instance in the case of equidistant points on a line, (5) leads to $e^{-\chi \frac{L(X)}{N}}$ rather than to $e^{-\chi L(X)}$. 
ii) Property (5) has also been derived in [3] at high temperature and arbitrary activity for lattice gases, by using the analyticity which is known in this domain and an explicit form of the truncated functions (at low activity).

iii) A stronger decrease of the type $e^{-\chi D(X)}$, where $D(X)$ is the diameter of the configuration $X$ has been derived in [4] for Ising ferromagnets at $\operatorname{Re} H \neq 0$, and for gases with non negative interaction potential when $\beta>0$ and $|z|$ is less than the radius of convergence of the Mayer $z$-expansion. It has also been proved in [1] as soon as there is a gap in the spectrum of the transfer matrix, for sufficiently small range. This coincides with $e^{-\chi L(X)}$ for lined up points but is still weaker otherwise. (The constant in front of $e^{-\chi D(X)}$ is also worse than $C^{N} N_{1} ! \ldots N_{p}$ !.)

\section{Results Proved in the Present Paper}

The methods and results of this paper are in a large measure extensions of methods and results of Ref. [3] and [4].

In Sections II to $\mathrm{V}$ we consider for simplicity lattice systems, the case of continuous systems being described in Section VI.

Some basic formulae and results on the expansions of the truncated correlations of lattice gases at low activity are described in Section II, at the end of which we mention various equalities between truncated functions ensuring i) that the results obtained for lattice gases imply corresponding results for spin lattices and ii) that the results obtained at low activity, resp. at $\operatorname{Re} H>H_{0}$, imply corresponding results at large activity, resp. at $\operatorname{Re} H<-H_{0}$.

In Section III, the strong cluster properties are directly derived at low activity i) in the Kirkwood-Salzburg region $|z|<e^{-2 \beta B-1} C(\beta)^{-1}$ and ii) for positive potentials, when $|z|$ is less than the radius of convergence of the Mayer $z$-expansion of the pressure.

In Section IV $\mathrm{A}$, the strong cluster properties are derived in larger regions of the complex $z$ space if the functions $\varrho_{\Lambda}^{T}(X)$ remain analytic and satisfy the bounds $\left|\varrho_{\Lambda}^{T}(X)\right| \leqq C^{N} N_{1} ! \ldots N_{p}$ ! in these regions for all sufficiently large $\Lambda$. It is shown that these properties hold in particular when the partition function $Z_{\Lambda}\left(\beta ; z_{1}, \ldots, z_{N}, z\right.$ ), (where $z_{1}, \ldots, z_{N}$ are specified activities of the points $x_{1}, \ldots, x_{N}$ ) does not vanish for any $z_{1}, \ldots, z_{N}, z$ in appropriate domains.

Using these results, the strong cluster properties are derived in Subsection B for general potentials and arbitrary activity, at high temperature. They are derived in Subsection $\mathrm{C}$ for ferromagnets i) at $\operatorname{Re} H \neq 0$ and arbitrary $\beta$, and ii) in the neighborhood of $H=0$ at high temperature. As a matter of fact they are derived, for any $\beta>0$, in any 
complex neighborhood of $H=0$ where $Z_{\Lambda}(\beta, H)$ does not vanish for all sufficiently large $\Lambda$.

In Section V, some general results on the links between analyticity and strong cluster properties, which extend those of Section IV A, are described. In particular, an equivalence is obtained between i) analyticity with respect to $z$ plus the bound $\left|\varrho_{\Lambda}^{T}\right| \leqq C^{N} N_{1} ! \ldots N_{p}$ ! and ii) the strong cluster properties.

Finally the results obtained for continuous gases are outlined in Section VI, as already announced.

\section{Preliminary Results (for Lattice Systems)}

The following expansions valid at low activity for lattice gases, at finite or infinite $\Lambda$, will be useful:

with

$$
\varrho_{\Lambda}^{T}(X ; \beta, z)=z^{N} \sum_{n \geqq 0} z^{n} C_{\Lambda, n}(X ; \beta)
$$

$$
\begin{aligned}
C_{A, n}(X ; \beta) & =\frac{1}{n !} \sum_{Y \in \Lambda^{n}} \varphi(X, Y ; \beta), \\
\varphi(X ; \beta) & =\sum_{\Gamma^{c}}^{X} \prod_{l \in \Gamma^{c}}\left(e^{-\beta \phi\left(d_{l}\right)}-1\right),
\end{aligned}
$$

where $\Sigma$ in (8) runs over all connected graphs $\Gamma^{c}$ joining the points of $X$ and $d_{l}$ is the length of line $l$. For a proof of these formulae see e.g. [5].

For a potential of finite range $\lambda$, the following crucial result can be checked in view of the definition of $L(X)$ given in the introduction:

$$
C_{A, n}(X ; \beta)=0 \text { for all } n<\frac{L(X)}{\lambda}-N+1 \text {. }
$$

We below denote $n_{0}(X)$ the first positive integer larger than (or equal to) $\frac{L(X)}{\lambda}-N+1$, and we may therefore write:

$$
\varrho_{\Lambda}^{T}(X ; \beta, z)=z^{N} \sum_{n \geqq n_{0}(X)} z^{n} \cdot C_{\Lambda, n}(X ; \beta) .
$$

In view of a well-known isomorphism [6], analogous results hold for spin systems, in which case $\varrho_{\Lambda}(X)$ is the distribution function of spin + at all sites of $X$. The truncated correlation functions $\left\langle\sigma_{x_{1}} \ldots \sigma_{x_{N}}\right\rangle_{\Lambda}^{T}$ are related to $\varrho_{\Lambda}^{T}(X)$ by:

$$
\begin{aligned}
\left\langle\sigma_{x}\right\rangle_{\Lambda}^{T} & =2 \varrho_{\Lambda}^{T}(X)-1 \\
\left\langle\sigma_{x_{1}} \ldots \sigma_{x_{N}}\right\rangle_{\Lambda}^{T} & =2^{N} \hat{\varrho}_{\Lambda}^{T}(X) \quad(N>1)
\end{aligned}
$$


where $\varrho(X)=\varrho(\hat{X})$, and $\hat{X}=\left(y_{1}, \ldots, y_{p}\right)$ denotes the set of positions occupied by $x_{1}, \ldots, x_{N} ; \hat{\varrho}(X)$ is identical to $\varrho(X)$ if all points $x_{1}, \ldots, x_{N}$ are different from each other. The bounds that we are going to derive will apply to $\varrho_{\Lambda}^{T}(X)$, but it can be checked ${ }^{2}$ that similar bounds can also be obtained for $\varrho_{\Lambda}^{T}(X)$, and therefore on $\left\langle\sigma_{x_{1}} \ldots \sigma_{x_{N}}\right\rangle_{\Lambda}^{T}$.

Finally, the following formulae hold under the symmetry $H \rightarrow-H$, where $H$ is the magnetic field, or correspondingly $z \rightarrow z^{-1} e^{\beta A}$ :

or

$$
\begin{aligned}
\hat{\varrho}_{\Lambda}^{T}(X,-H) & =(-1)^{N} \hat{\varrho}_{\Lambda}^{T}(X, H) \\
\left\langle\sigma_{x_{1}} \ldots \sigma_{x_{N}}\right\rangle_{\Lambda}^{T}(-H) & =(-1)^{N}\left\langle\sigma_{x_{1}} \ldots \sigma_{x_{N}}\right\rangle_{\Lambda}^{T}(H) .
\end{aligned}
$$

Therefore, the strong cluster properties in some domain will imply analogous strong cluster properties in the domains obtained by the above transformations.

\section{Strong Cluster Properties at Low Activity}

A. Results in the Kirkwood-Salsburg Domain

Let $C(\beta)=\sum_{x \in Z^{v}}\left|e^{-\beta \phi(x)}-1\right|$, and $r(\beta)=e^{-2 \beta B-1} \cdot C(\beta)^{-1}$ where $B$ is the stability constant.

Theorem 1. For $|z|<r(\beta)$, the following bound, where $\alpha$ is an arbitrary real number such that $1<\alpha<\frac{r(\beta)}{|z|}$, and $\Lambda$ is finite or infinite, holds:

$$
\left|\varrho_{\Lambda}^{T}(X ; \beta, z)\right| \leqq N_{1} ! \ldots N_{p} ! \frac{e^{-2 \beta B} r(\beta)}{1-\alpha \frac{|z|}{r(\beta)}} \frac{1}{(\alpha-1)^{N}}\left(\alpha \cdot \frac{|z|}{r(\beta)}\right)^{\frac{L(X)}{\lambda}} .
$$

This bound is clearly a strong cluster property of type (4), with $\chi=\frac{1}{\lambda} \log \frac{r(\beta)}{\alpha|z|}$.

Proof. The following inequality on the Ursell function $\varphi$ holds [5]:

$$
\sum_{x_{2}, \ldots, x_{m}}\left|\varphi\left(x_{1}, x_{2}, \ldots, x_{m} ; \beta\right)\right| \leqq(m-1) ! \frac{e^{-2 \beta B}}{r(\beta)^{m-1}} .
$$

Since:

$$
\sum_{x_{2}, \ldots, x_{N}}\left|C_{A, n}(X ; \beta)\right| \leqq \sum_{\substack{x_{2}, \ldots, x_{N} \\ y_{1}, \ldots, y_{n}}}|\varphi(X, Y ; \beta)|
$$

${ }^{2}$ This is not completely trivial in the case of identical points since $\hat{\varrho}_{A}^{T}(X)$ is truncated with respect to all $N$ variables $x_{1}, \ldots, x_{N}$ and is therefore different from $\varrho_{\Lambda}^{T}(\hat{X})$. 
it is readily checked that:

$$
\left|C_{\Lambda, n}(X ; \beta)\right| \leqq N_{1} ! \ldots N_{p} ! \frac{(N+n-1) !}{n !(N-1) !} \frac{e^{-2 \beta B}}{r(\beta)^{N+n-1}} .
$$

Then, by using the identity:

$$
\sum_{n \geqq n_{0}} t^{n} \frac{(k+n) !}{n !}=\frac{d^{k}}{d t^{k}}\left(\frac{t^{n_{0}+k}}{1-t}\right)
$$

and a Cauchy formula, we get for $1<\alpha<\frac{1}{|t|}$

$$
\sum_{n \geqq n_{0}}|t|^{n} \frac{(k+n) !}{n !} \leqq k ! \frac{(\alpha|t|)^{n_{0}}}{1-\alpha|t|}\left(\frac{\alpha}{\alpha-1}\right)^{k} .
$$

The inequality (13) is readily derived from (10) by making use of this formula with $t=\frac{\alpha z}{r(\beta)}$.

\section{B. Results for Positive Potentials (or Antiferromagnets)}

Let $\mathscr{R}(\beta)$ be the radius of convergence of the Mayer $z$-expansion of the pressure $p$. Then:

Theorem 2. For positive potentials, the following bounds hold at $|z|<\mathscr{R}(\beta)$ :

$$
\left|\varrho_{\Lambda}^{T}(X ; \beta, z)\right| \leqq C_{\gamma} N_{1} ! \ldots N_{p} ! N\left(\frac{1}{\alpha-1}\right)^{N}\left(\frac{\alpha \gamma|z|}{\mathscr{R}(\beta)}\right)^{\frac{L(X)}{\lambda}}
$$

where $\alpha$ and $\gamma$ are arbitrary real numbers such that $\gamma>1,1<\alpha<\frac{\mathscr{R}(\beta)}{\gamma|z|}$, and where $C_{\gamma}=\operatorname{Max}|\beta p(\beta ; z)|$.

$$
|z|<\frac{\mathscr{R}(\beta)}{\gamma}
$$

Proof. The following result holds (for general potentials):

$$
\sum_{x_{2}, \ldots, x_{m} \in \Lambda} \varphi\left(x_{1}, x_{2}, \ldots, x_{m} ; \beta\right)=\left(\frac{\partial}{\partial z}\right)^{m}\left[\beta p_{\Lambda}(\beta, z)\right]_{z=0}
$$

where $\Lambda$ is finite or infinite.

Now, for positive potentials, it turns out that [7]

$$
\varphi\left(x_{1}, \ldots, x_{m} ; \beta\right)=(-1)^{m+1}\left|\varphi\left(x_{1}, \ldots, x_{m} ; \beta\right)\right|
$$


and therefore:

$$
\sum_{x_{2}, \ldots, x_{m} \in \Lambda}\left|\varphi\left(x_{1}, x_{2}, \ldots, x_{m} ; \beta\right)\right| \leqq C_{\gamma} \frac{\gamma^{m}}{\mathscr{R}(\beta)^{m}} m ! .
$$

The proof of (16) then follows by the same methods as in Subsection A.

\section{Strong Cluster Properties Derived from Results on the Zeroes of the Partition Function}

\section{A. General Theorems}

The strong cluster properties can also be derived from formula (10) in larger domains by making use of the following theorem inspired from Ref. [3] and [4].

Theorem 3. Let $\mathscr{D}$ be an open connected complex domain in z-space containing $z=0$, such that i) $\varrho_{\Lambda}^{T}(X ; \beta, z)$ remains analytic in $\mathscr{D}$ at some given $\beta>0$, for sufficiently large finite $\Lambda$ and all configurations $X$ and ii) $\varrho_{\Lambda}^{T}(X)$ moreover satisfies the bound:

$$
\left|\varrho_{\Lambda}^{T}(X ; \beta, z)\right|<C^{N} N_{1} ! \ldots N_{p} !
$$

where $C$ is independent of $\Lambda, X$ and of $z$ in $\mathscr{D}$.

Then the following strong cluster properties hold for $\Lambda$ finite or infinite and $z$ in $\mathscr{D}$ :

$$
\left|\varrho_{\Lambda}^{T}(X ; \beta, z)\right| \leqq C^{N} \frac{N_{1} ! \ldots N_{p} !}{1-|t(z)|}|t(z)|^{\frac{L(X)}{\lambda}}
$$

where $|t(z)|<1 ; t$ is a conformal mapping of $\mathscr{D}$ onto the interior of the unit circle such that $t(0)=0$.

Proof. Using the conformal mapping $z \rightarrow t(z)$, a series expansion analogous to (10) can be written in $t$-space:

$$
\varrho_{\Lambda}^{T}(X ; \beta, t)=t^{N} \cdot \sum_{n \geqq n_{0}(X)} t^{n} \gamma_{\Lambda, n}(X ; \beta) .
$$

Owing to the analyticity of $\varrho_{A}^{T}$ with respect to $t$ in the region $|t|<1$, we get $\left|\gamma_{\Lambda, n}(X ; \beta)\right|=\frac{1}{2 \pi}\left|\oint \frac{\varrho_{\Lambda}^{T}(X ; \beta, t)}{t^{N+n+1}} d t\right|$.

Using (20) and integrating over a circle of radius as near as wanted to 1 , one gets:

$$
\left|\gamma_{\Lambda, n}(X ; \beta)\right| \leqq C^{N} \cdot N_{1} ! \ldots N_{p} !
$$


from which (21) immediately follows. (The limit of infinite $\Lambda$ is obtained as a consequence of Vitali's theorem since the convergence is known at small $|z|$.)

Remark. We notice that the best value of $|t(z)|$ for a given $z$ depends on the precise domain of analyticity of $\varrho_{\Lambda}^{T}(X ; \beta, z)$ with respect to $z$ and therefore on the reciprocal temperature $\beta$.

Besides the cases already studied in Section III, the hypotheses of Theorem 3 can also be checked in other situations, in particular by using the following result:

Theorem 4. Let $Z_{\Lambda}\left(\beta ; z_{1}, \ldots, z_{N}, z\right)$ be the partition function, where $z_{1}, \ldots, z_{N}$ are specified activities of points $x_{1}, \ldots, x_{N}$, and let $\mathscr{D}^{\prime}$ be an open complex domain in z-space such that for some $\beta>0$ $Z_{\Lambda}\left(\beta ; z_{1}, \ldots, z_{N}, z\right) \neq 0$ for sufficiently large finite $\Lambda$, when $z_{1} \in \mathscr{D}^{\prime}, \ldots, z_{N}$ $\in \mathscr{D}^{\prime}, z \in \mathscr{D}^{\prime}$.

Then $\varrho_{\Lambda}^{T}(X ; \beta, z)$ is analytic in $\mathscr{D}^{\prime}$ and satisfies the bound $\left|\varrho_{\Lambda}^{T}(X ; \beta, z)\right|<C^{N} N_{1} ! \ldots N_{p}$ !, where $C$ is independent of $\Lambda$, and $z$ in any set $K \subset \mathscr{D}^{\prime}$ whose distance to the boundary of $\mathscr{D}^{\prime}$ is strictly positive, and also of $N$ and $X$ if $\mathscr{D}^{\prime}$ is.

Proof. In the case when the points $x_{1}, \ldots, x_{N}$ occupy only $p$ different positions $y_{1}, \ldots, y_{p}$, we shall denote by $z_{1}, \ldots, z_{p}$ the corresponding activities at $y_{1}, \ldots, y_{p}$.

The following formula holds:

$$
\begin{aligned}
\frac{\varrho_{\Lambda}^{T}(X ; \beta, z)}{z^{N}} & =\left.\frac{\partial^{N_{1}}}{\partial z_{1}^{N_{1}} \ldots \frac{\partial^{N_{p}}}{\partial z_{p}^{N_{p}}}} \log Z_{\Lambda}\left(\beta ; z_{1}, \ldots, z_{p}, z\right)\right|_{z_{1}=\ldots=z_{p}=z} \\
& =\frac{N_{1} ! \ldots N_{p} !}{(2 i \pi)^{p}} \oint_{\substack{\left|z_{i}-z\right|=\alpha \\
i=1, \ldots, p}} \frac{\log Z_{\Lambda}\left(\beta ; z_{1}, \ldots, z_{p}, z\right)}{\left(z_{1}-z\right)^{N_{1}+1} \ldots\left(z_{p}-z\right)^{N_{p}+1}} d z_{1} \ldots d z_{p}
\end{aligned}
$$

where $z \in \mathscr{D}^{\prime}$, and $\alpha$ is choosen such that the circles $\left|z_{i}-z\right|=0$ also belong to $\mathscr{D}^{\prime}$.

By writing $Z_{\Lambda}\left(\beta ; z_{1}, \ldots, z_{p}, z\right)$ in the form:

$$
\begin{aligned}
Z_{\Lambda}\left(\beta ; z_{1}, \ldots, z_{p}, z\right) & =P_{\Lambda}\left(\beta ; z_{2}, \ldots, z_{p}, z\right)+z_{1} Q_{\Lambda}\left(\beta ; z_{2}, \ldots, z_{p}, z\right) \\
& =Q_{\Lambda}\left(\beta ; z_{2}, \ldots, z_{p}, z\right)\left[z_{1}-\zeta_{1, \Lambda}\left(\beta ; z_{2}, \ldots, z_{p}, z\right)\right]
\end{aligned}
$$

formula (24) can also be written:

$$
\begin{aligned}
& \frac{\varrho_{\Lambda}^{T}(X ; \beta, z)}{z^{N}}=\frac{\left(N_{1}-1\right) ! N_{2} ! \ldots N_{p} !}{(2 i \pi)^{p}} \\
& \cdot \oint_{\substack{\left|z_{i}-z\right|=\alpha \\
i=1, \ldots, p}} \frac{d z_{1} \ldots d z_{p}}{\left[z_{1}-\zeta_{1, \Lambda}\left(\beta ; z_{2}, \ldots z_{p}, z\right)\right]\left(z_{1}-z\right)^{N_{1}}\left(z_{2}-z\right)^{N_{2}+1} \ldots\left(z_{p}-z\right)^{N_{p+1}}}
\end{aligned}
$$

from which the theorem easily follows. 


\section{B. Strong Cluster Properties at High Temperature and Arbitrary Activity}

It follows from Ref. [8], that at sufficiently high temperatures the partition function $Z_{\Lambda}\left(\beta ; z_{1}, \ldots, z_{N}, z\right)$ of a lattice gas has no zeroes when all activities $z_{1}, \ldots, z_{N}, z$ lie in some open domain of the complex $z$-space containing the positive real axis $z \geqq 0$. As a consequence of Theorems 3 and 4 , the strong cluster properties are therefore proved in this domain.

\section{Strong Cluster Properties for Ferromagnets at $\operatorname{Re} H \neq 0, \beta$ Arbitrary and at $H=0$ for Sufficiently High Temperature}

In view of Lee and Yang's lemma [9], $Z_{\Lambda}\left(\beta ; H_{1}, \ldots, H_{p}, H\right)$, where $H$, resp. $H_{1}, \ldots, H_{p}$, is the magnetic field, resp. the magnetic field at points $y_{1}, \ldots, y_{p}$, is different from zero when $\operatorname{Re} H>0, \operatorname{Re} H_{1}>0, \ldots, \operatorname{Re} H_{p}>0$.

The strong cluster properties of the truncated correlations $\left\langle\sigma_{x_{1}} \ldots \sigma_{x_{N}}\right\rangle_{\Lambda}^{T}$ at $\operatorname{Re} H>0$ and $\operatorname{Re} H<0$ follow again from Theorems 3 and 4 and from the remarks at the end of Section II.

Now the result of Section $\mathrm{B}$, which is valid for general potentials also implies the strong cluster properties for $\operatorname{Re} H$ arbitrary and small $|\operatorname{Im} H|$, at high temperature.

As a matter of fact, in the specific case of ferromagnets the following more precise result is obtained: if for some $\beta>0$, and sufficiently large $\Lambda$, $Z_{\Lambda}(\beta ; H) \neq 0$ when $H$ belongs to a complex neighborhood $\mathscr{N}$ of $H=0$, the strong cluster properties also hold in this neighborhood.

Proof. The result is already known at all points of $\mathscr{N}$ for which $\operatorname{Re} H \neq 0$ (see above). Now, Theorem 3 of Ref. [10] ensures the existence of an appropriate domain $\mathscr{N}^{\prime}$ containing all points of $\mathscr{N}$ for which $\operatorname{Re} H=0$ and such that $Z_{\Lambda}\left(\beta ; H_{1}, \ldots, H_{p}, H\right)$ is also different from zero for $H_{1}, \ldots, H_{p}, H$ in this domain.

Remark. By applying some results of Ref. [10] and [11], together with Theorems 3 and 4, further results can also be obtained in the case of antiferromagnets with nearest neighbor interactions, and possibly in other situations.

\section{Analyticity and Strong Cluster Properties: Further General Results}

We first give a result which extends Theorem 3 of Section IV. It may concern the gas and liquid phases and in fact applies to points $\left(\beta_{0}, z_{0}\right)$ where "the correlation functions are analytically linked to the KirkwoodSalsburg domain". 
Theorem 5. Let $\left(\beta_{0}, z_{0}\right)$ be a point of the real $(\beta, z)$ plane. Suppose that there exists an analytic mapping $t \rightarrow(\beta(t), z(t))$ of the unit circle $|t|<1$ in $\mathbb{C}^{2}$ with

1) $\beta(0)>0, z(0)=0$

2) $\beta\left(t_{0}\right)=\beta_{0}, z\left(t_{0}\right)=z_{0}$ for some $t_{0},\left|t_{0}\right|<1$,

such that the correlations $\varrho_{\Lambda}^{T}(X ; t)=\varrho_{\Lambda}^{T}(X ; \beta(t), z(t))$ extend analytically with respect to $t$ in the unit circle $|t|<1$ for any $X=\left(x_{1}, \ldots, x_{N}\right)$ and finite $\Lambda$. Suppose that moreover the following bound holds for $|t|<1$ :

$$
\left|\varrho_{A}^{T}(X ; \beta(t), z(t))\right|<A_{N}
$$

where $A_{N}$ is independent of $t, \Lambda$ ( and may depend on $N_{1}, \ldots, N_{p}$ ).

Then the following strong decrease is obtained for $\Lambda$ finite or infinite:

$$
\left|\varrho_{\Lambda}^{T}\left(X ; \beta_{0}, z_{0}\right)\right|<A_{N} \frac{\left|t_{0}\right|^{\frac{L(X)}{\lambda}}}{1-\left|t_{0}\right|} .
$$

We note that this bound is a strong cluster property of type (4) if $A_{N}$ is of the form $C^{N} \cdot N_{1} ! \ldots N_{p}$ !.

Proof. It follows from the hypothesis that the series expansion

$$
\varrho_{\Lambda}^{T}(X ; t)=\sum_{n \geqq 0} a_{n}(X) \cdot t^{n}
$$

is absolutely convergent for $|t|<1$.

For $|t|$ small enough $(\beta(t), z(t))$ belongs to the Kirkwood-Salsburg domain of analyticity with respect to both variables $\beta$ and $z$. Besides:

$$
\varrho_{\Lambda}^{T}(X ; t)=z(t)^{N} \sum_{n \geqq n_{0}(X)} z(t)^{n} C_{\Lambda, n}(X ; \beta(t)) .
$$

Using the analyticity of $C_{\Lambda, n}(X ; \beta)$ with respect to $\beta$ in a neighbourhood of $\beta(0)$, and the assumptions of the theorem, we have for $|t|$ small enough, and then also for $|t|<1$ :

$$
\varrho_{\Lambda}^{T}(X ; t)=t^{N} \sum_{n \geqq n_{0}(X)} t^{n} \gamma_{\Lambda, n}(X) .
$$

For the same reasons as in Theorem 2, it therefore follows that:

$$
\left|\varrho_{\Lambda}^{T}(X ; t)\right| \leqq A_{N} \cdot \frac{|t|^{N+n_{0}(X)}}{1-|t|}
$$

which reduces to (28) when $t=t_{0}$.

Finally it seems interesting to note the following equivalence theorem:

Theorem 6. Let $\left(\beta_{0}, z_{0}\right)$ be a real point of the $(\beta, z)$ plane. Then the two following properties are equivalent:

1. There exist a complex neighbourhood $\mathcal{O}$ of $\left.] 0, z_{0}\right]$ in complex $z$-space and a constant $C$ such that for any (finite) $\Lambda$ and any $X=\left(x_{1}, \ldots, x_{N}\right)$, 
$\varrho_{\Lambda}^{T}\left(X ; \beta_{0}, z\right)$ is analytic with respect to $z$ in $\mathcal{O}$ and satisfies:

$$
\left|\varrho_{\Lambda}^{T}\left(X ; \beta_{0}, z\right)\right|<C^{N} \cdot N_{1} ! \ldots N_{p} !, \quad \forall z \in \mathcal{O} .
$$

2. There exist two (positive) constants $C^{\prime}$ and $\chi$ such that for any (finite) $\Lambda$ and any $X=\left(x_{1}, \ldots, x_{N}\right)$, the following strong cluster property holds for all real $\left.z \in] 0, z_{0}\right]$ :

$$
\left|\varrho_{\Lambda}^{T}\left(X ; \beta_{0}, z\right)\right|<C^{\prime N} N_{1} ! \ldots N_{p} ! e^{-\chi L(X)} .
$$

Proof. The proof that 1 . implies 2. is a straightforward consequence of Theorem 3.

The proof that 2. implies the analyticity of the correlation functions in a complex neighbourhood of $\left.] 0, z_{0}\right]$ is a consequence of [1]. We give here a direct derivation which yields at the same time the bound (31); as a matter of fact a stronger result will be obtained, namely that the bound (32) at a real point $(\beta, z)$ implies an analogous bound in some complex neighbourhood in $z$ of this point.

We start from the formula, for real points $(\beta, z)$ :

$$
\frac{\partial^{n}}{\partial z^{n}}\left(\frac{\varrho_{\Lambda}^{T}(X ; \beta, z)}{z^{N}}\right)=\frac{1}{z^{N+n}} \sum_{Y \in \Lambda^{n}} \varrho_{\Lambda}^{T}(X, Y ; \beta, z)
$$

which is obtained from (24).

Besides, we have the two following inequalities:

$$
L(X, Y) \geqq(1-\varepsilon) L(X)+\varepsilon L\left(x_{1}, Y\right) \quad \text { with } \quad 0<\varepsilon<1
$$

and

$$
M_{1} ! \ldots M_{q} ! \leqq 2^{N+n} N_{1} ! \ldots N_{p} ! n_{1} ! \ldots n_{r} !
$$

where the $M_{i}, N_{j}$ and $n_{k}$ are respectively the numbers of identical points of $(X, Y), X$ and $Y$.

These inequalities, together with (32), lead to:

$$
\left|\varrho_{\Lambda}^{T}(X, Y ; \beta, z)\right| \leqq\left(2 C^{\prime}\right)^{N} N_{1} ! \ldots N_{p} ! e^{-(1-\varepsilon) \chi L(X)}\left(2 C^{\prime}\right)^{n} n_{1} ! \ldots n_{r} ! e^{-\varepsilon \chi L\left(x_{1}, Y\right)}
$$

Since the bound (4) is better than the bound (1), we can use the result of [1] on the integrals of the right-hand side of (1), and the Eqs. (33) and (36) yield:

$$
\left|\frac{\partial^{n}}{\partial z^{n}}\left(\frac{\varrho_{\Lambda}^{T}(X ; \beta, z)}{z^{N}}\right)\right| \leqq\left(\frac{2 C^{\prime}}{z}\right)^{N} N_{1} ! \ldots N_{p} ! e^{-(1-\varepsilon) \chi L(X)}\left(\frac{A(\varepsilon)}{z}\right)^{n} n !
$$

where $A(\varepsilon)$ depends only on $\varepsilon\left[\right.$ and $\left.\left(\beta_{0}, z_{0}\right)\right]$. 
Now (29) implies for $\left.\left.\left|z^{\prime}-z\right|<\frac{z}{A(\varepsilon)}, z \in\right] 0, z_{0}\right]$

$$
\left|\varrho_{\Lambda}^{T}\left(X ; \beta, z^{\prime}\right)\right| \leqq\left(2 C^{\prime}\right)^{N} N_{1} ! \ldots N_{p} ! \frac{1}{1-A(\varepsilon) \frac{\left|z^{\prime}-z\right|}{z}} e^{-(1-\varepsilon) \chi L(X)}
$$

which is the stronger result announced. It clearly implies property 1. of Theorem 4, with $\mathcal{O}=\bigcup_{\left.z \in] 0, z_{0}\right]} \mathscr{B}\left(z ; \frac{z}{\mathrm{~A}(\varepsilon)}\right)$, where $\mathscr{B}\left(z ; \frac{z}{\mathrm{~A}(\varepsilon)}\right)$ is the disk of center $z$ and radius $\frac{z}{A(\varepsilon)}$ in complex $z$-plane.

\section{The Strong Cluster Properties for Continuous Systems}

In this section, we outline how the Theorems 1-3, 5, 6 apply also, with slight modifications, for continuous systems with stable potentials.

Eqs. (6) and (8) to (10) hold again for continuous systems with (7) replaced by

$$
C_{\Lambda, n}(X ; \beta)=\frac{1}{n !} \int_{\Lambda^{n}} \varphi(X, Y ; \beta) d Y
$$

and now:

$$
C(\beta)=\int_{\mathbb{R}^{v}}\left|e^{-\beta \phi(x)}-1\right| d x .
$$

Let us introduce a partition of $\mathbb{R}^{v}$ by the cells of any given lattice, and consider the following averaged truncated correlation functions:

$$
\varrho^{T}\left(x_{1}, \ldots, x_{n}\right)=\int \varrho^{T}\left(y_{1}, \ldots, y_{n}\right) \varphi_{x_{1}}\left(y_{1}\right) \ldots \varphi_{x_{n}}\left(y_{n}\right) d y_{1} \ldots d y_{n}
$$

where $\varphi_{x_{i}},(i=1, \ldots, n)$ is the characteristic function of the cell indexed by $x_{i} \in Z^{v}$.

Theorems 1 and 2 now apply to the functions $\varrho_{\Lambda}^{T}(X ; \beta, z)$.

Theorems 3, 5 and 6 apply both to $\varrho_{\Lambda}^{T}(X ; \beta, z)$ and $\tilde{\varrho}_{\Lambda}^{T}(X ; \beta, z)$.

As a matter of fact, more general test functions can be used in (40). Then the results still hold, with $N_{1} ! \ldots N_{p}$ ! replaced by a multiplicity factor depending on the support of the test function.

\section{Conclusion}

As a conclusion, we would like to mention the following questions:

1. Theorem 6 states the equivalence between the strong cluster properties and analyticity with respect to $z$, plus the bounds $\left|\varrho_{\Lambda}^{T}(X)\right|$ $<C^{N} N_{1}$ ! ... $N_{p}$ !. We do not know as yet whether this last condition can be removed. 
2. In paper [1], strong cluster hypotheses on "generalized" truncated functions have also been introduced and have been shown to imply analyticity with respect to $\beta$ and possibly other coupling constants. Some converse assertions seem to be true, but there are some problems which are being studied.

3. Concerning possible proofs of strong cluster properties when analyticity is not already known or assumed, and which would therefore give new information on analyticity, we do not know as yet what are the actual possibilities in this domain.

4. In a recent work [12], J. L. Lebowitz and O. Penrose have shown that if $Z_{\Lambda}(\beta, z) \neq 0$ for all sufficiently large $\Lambda$ and $z$ in an open connected region $\mathscr{D}$ which contains $z=0$, then decrease properties of the type mentioned in (5) (or of the type $e^{-\chi D}$ ) follow for all $z$ in $\mathscr{D}$ : this is obtained by proving the existence of a gap of the transfer matrix. This result seems related in some way to Theorems 3,4 .

5. A coming paper will treat the case of infinite range potentials.

We are very grateful to Professors G. Gallavotti, J. Lascoux, E. H. Lieb, J. Slawny and more particularly J.L. Lebowitz and D. Ruelle for helpful discussions or correspondence.

\title{
References
}

1. Duneau, M., Iagolnitzer, D., Souillard, B.: Commun. math. Phys. 31, 191 (1973)

2. Lebowitz, J. L.: Commun. math. Phys. 28, 313 (1972)

3. Gallavotti, G., Miracle-Sole, S.: Commun. math. Phys. 12, 269 (1969)

4. Lebowitz, J.L., Penrose, O.: Commun. math. Phys. 11, 99 (1968)

5. Ruelle, D.: Statistical mechanics, rigourous results. New York: Benjamin 1969

6. Yang, C. N., Lee, T.D.: Phys. Rev. 87, 404 (1952)

7. Groeneveld, J.: Phys. Letters 3, 50 (1962)

8. Ruelle, D.: Phys. Rev. Letters 26, 303 (1971)

9. Lee, T.D., Yang, C. N.: Phys. Rev. 87, 410 (1952)

10. Lieb,E.H., Ruelle, D.: J. Math. Phys. 13, 781 (1972)

11. Ruelle, D.: Commun. math. Phys. 31, 265 (1973)

12. Lebowitz, J.L., Penrose, O.: Decay of correlation, preprint

Communicated by G. Gallavotti

\author{
M. Duneau \\ B. Souillard \\ Centre de Physique Théorique \\ de l'Ecole Polytechnique \\ 17, rue Descartes \\ F-75230 Paris Cédex 05, France \\ D. Iagolnitzer \\ Service de Physique Théorique \\ Centre d'Etudes Nucléaires de Saclay \\ F-91190 Gif-sur-Yvette, France
}

